\title{
Precise Prediction of Near-Net-Shape HIP Components through DEM and FEM Modelling
}

\author{
Yuanbin Deng ${ }^{1, \mathrm{a}}{ }^{*}$, Anke Kaletsch $^{1, \mathrm{~b}}$, Alexander Bezold ${ }^{1, \mathrm{c}}$ and \\ Christoph Broeckmann ${ }^{1, d}$ \\ ${ }^{1}$ RWTH Aachen University, Institute for Materials Applications in Mechanical Engineering \\ (IWM), Augustinerbach 4, D-52062, Aachen, Germany \\ ay.deng@iwm.rwth-aachen.de, ba.kaletsch@iwm.rwth-aachen.de, \\ ca.bezold@iwm.rwth-aachen.de, ${ }^{\mathrm{d}} \mathrm{c}$.broeckmann@iwm.rwth-aachen.de
}

Keywords: Hot Isostatic Pressing (HIP), Discrete-Element-Method (DEM), Powder Filling, Finite-Element-Method (FEM), Powder Densification, Modelling, Simulation

\begin{abstract}
In Hot Isostatic Pressing (HIP) of metal powder, anisotropic shrinkage of the capsule induced by inhomogeneity of the initial powder filling density determines the reproducible realization of small geometrical allowances. This becomes a detrimental factor in the manufacturing of near-net-shape components due to their high requirements for the final shape accuracy. This challenge can be solved by precisely predicting and controlling the shrinkage with respect to the filling density via numerical simulation. Using Discrete-Element-Method (DEM), a three-dimensional initial powder density distribution of the whole component is simulated. After being validated by experimental results from metallographic examination, the calculated powder density distribution is mapped to a Finite-Element (FE) model. An in-house developed user defined material (UMAT) Subroutine, which considers both instantaneous plasticity at lower temperatures and rate dependent plasticity at higher temperatures, is utilized for the simulation with ABAQUS. The preliminary experimental validation using lab scale component reveals that the shrinkage induced shape changes during HIP can be accurately predicted by iterative simulations. Furthermore, the influences of local density distribution during HIP are also investigated. In summary, the developed simulation method demonstrates high accuracy in HIP component shape prediction. Therefore, the method can be easily used for designing HIP capsules for large and complex components.
\end{abstract}

\section{Introduction}

Hot Isostatic Pressing of encapsulated metal powder (PM HIP) is a modern manufacturing process to produce complex and highly specified components using a wide range of metals and alloys. The major advantage of PM HIP is the possibility to produce large and massive near-netshape (NNS) metal components with complex shapes and excellent mechanical properties. In theory, hot isostatically pressed components should have isotropic shrinkage under isostatic pressure load. However, in practice this is usually not the case. Non-uniform shrinkage of components and even high distortion have been observed. This leads to high cost of post processing and long delivery times.

Theoretically, the three main factors which can lead to the distortion of the final shape of HIPed components are ${ }^{[1-5]}$ : inhomogeneous powder density distribution in the capsule, temperature gradients in the HIP unit, and imperfection of capsule and powder materials. In previous work ${ }^{[6]}$, it has been found that the inhomogeneous initial density distribution of the metal powder in the capsule is the dominating factor that causes the anisotropic shrinkage and distortion of the final component shape (Figure 1). 

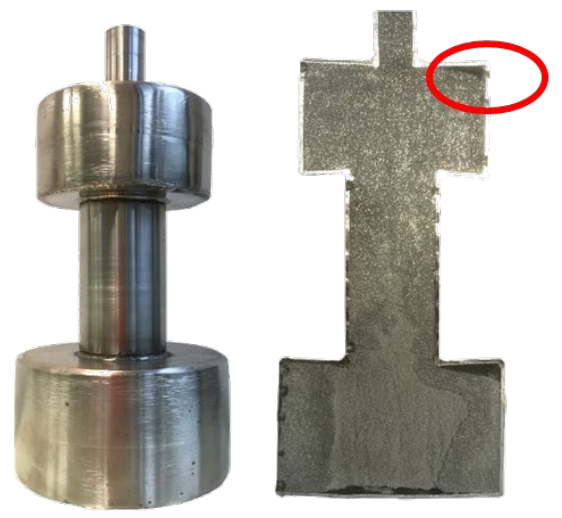

(a)
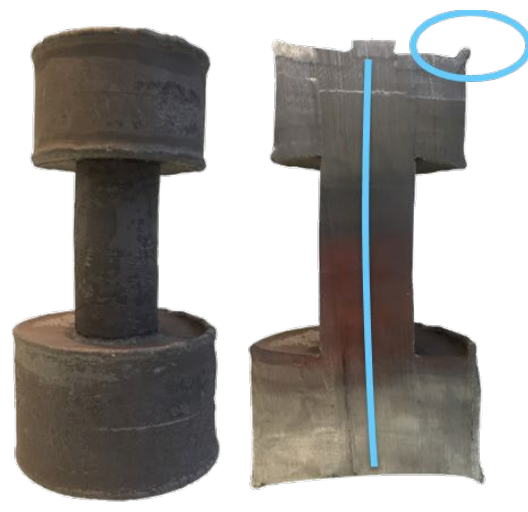

(b)

Figure 1: (a) Welded Capsule (SS304) filled with powder (SS316L, CARPENTER, Melt 500, $d=140 \mu \mathrm{m})$ prior to HIP, the red cycle shows the zone, which has a poor filling density; (b) Capsule (SS304) filled with powder (SS316L) consolidated to full density after HIP, the blue lines show the bending of capsule and anisotropic deformation

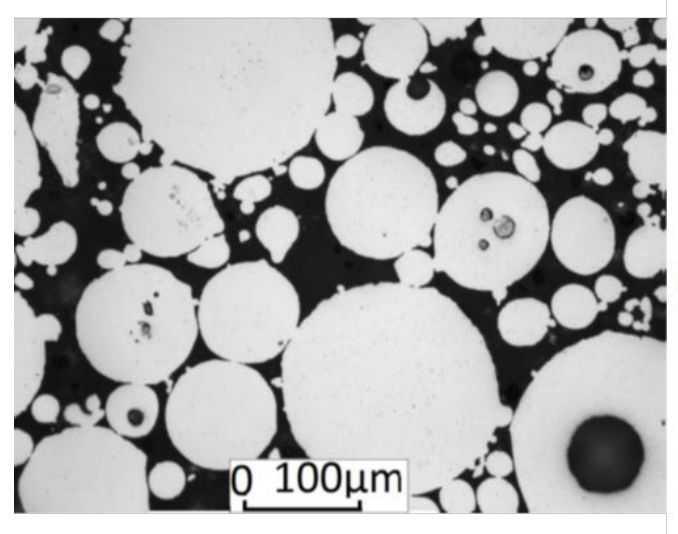

(a)

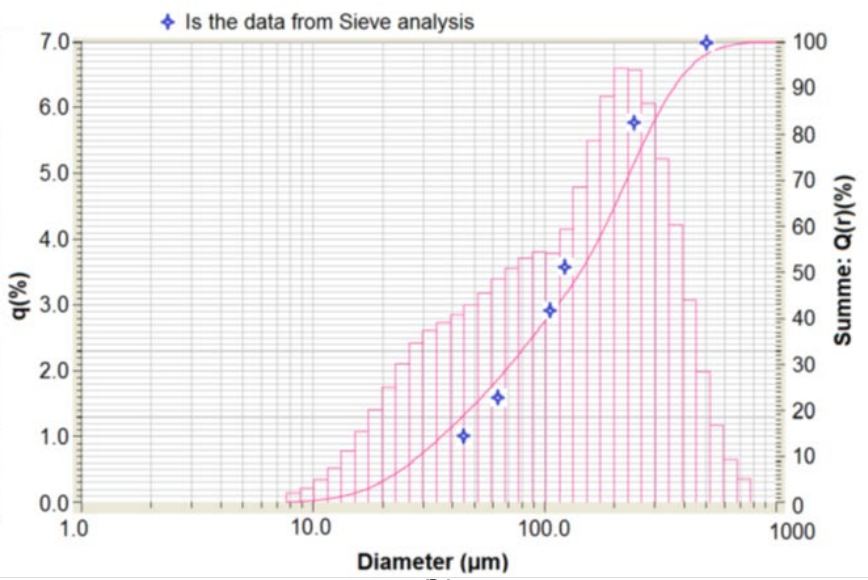

(b)

Figure 2: (a) A microstructure of powder prior to HIP (Light optical micrograph); (b) Powder particle size distribution (Laser Scattering Particle Size Distribution Analyzer LA 950)

The powder used in this study has a mean particle size of $140 \mu \mathrm{m}$ as shown in Figure 2. The results were obtained from dry measurement done by the Laser-Scattering Particle Size Distribution Analyzer Type Horiba LA-950.

This work focuses on the numerical determination of the powder density distribution in the capsule prior to the HIP. Combining the Discrete-Element-Method (DEM) and the FiniteElement-Method (FEM) on the one hand, the powder density distribution is studied numerically. On the other hand, the influences of the local density distribution during HIP are also investigated via simulation. This reduces the need for the often-costly prototypes and trial-anderror processes that are nowadays inherent to capsule design and final shape prediction. 


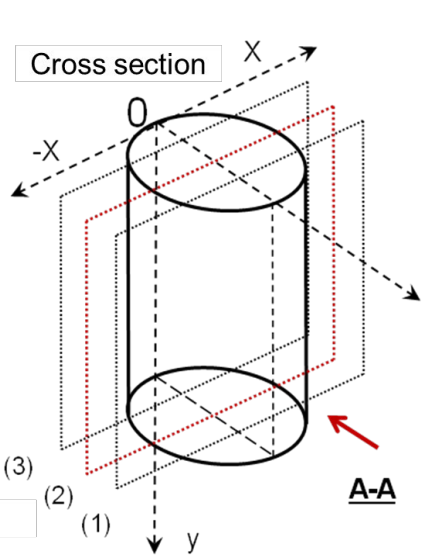

(a)

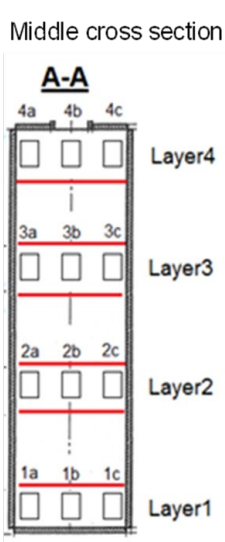

(b)

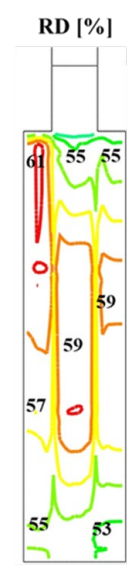

(c)

Figure 3: Arrangement of measured points for Image Analysis; (a) several cross sections were analysed; (b) sketch of measured points (Sub-windows) for the cross section along a radial-axial plane; (c) example of a relative density distribution at the middle cross

section of a complex HIPed component. (RD: Relative density)

\section{State of the technology}

\section{"Image Analysis" - Experimental Determination}

Previous investigation ${ }^{[7]}$ showed that the initial powder density distribution in the capsule is strongly influenced by the filling procedure and the precompaction process prior to HIP. To analyse the density distribution in the capsule, the powder particles must be "frozen" in their positions after filling and pre-compaction. Therefore, a pressure-less pre-sintering step, which induces very limited overall shrinkage, was applied to build up weak connections between individual particles. After this pressure-less presintering at a temperature close to the HIP temperature for $30 \mathrm{~min}$, the arrangement of the powder particles in the capsule was fixed. In this state, the capsule was cut along several planes

(Figure 3(a) and (b)) to obtain samples for the metallographic preparation. Based on the developed "Image Analysis" method, the micrographs of the samples were captured, stored and sorted. The resolution and contrast of all the images were controlled and adjusted to distinguish between porosity and powder particles. The obtained images were digitalized and then converted to the relative density values. These values were then imported into the simulation model (Figure 3 (c)) using an in-house written MATLAB script. However, the determination of the local density distribution is relative time-consuming. Only the relative density on the selected cross planes were measured and the values were extrapolated on the rest areas. The deviation between the determined and real filling density was measured and less than $5 \%$. Due to these limitations, a numerical method was developed to calculate and determine the powder density distribution inside filled capsule.

\section{Methodology}

\section{Discrete-Element-Method (DEM) - Numerical Determination}

In recent years, due to the advancement in computer processing speed, numerical simulation of granular flow becomes increasingly effective as an alternative tool to study the behaviours of powder flow, filling and the pre-consolidation process. Since a granular system is composed of individual particles and each particle moves independently of each other, it is difficult to predict the behaviour of a granular system using continuum mechanical models. In this context, the discrete approach developed for particle scale numerical modelling of granular materials has become a powerful and reliable tool. It is considered as an alternate to the continuum approach. This discrete approach is generally referred to as Discrete-Element-Method (DEM). 
DEM is a particle method based on Newton's laws of motion. Particles can move with three degrees of freedom (three for translational movement). The particles are defined as soft-particles with consideration of material deformation. During the calculation, a contact model is applied to calculate the contact forces between individual particles. Furthermore, external forces such as gravity or external pressure can be introduced into the simulation as well ${ }^{[8]}$. All the simulation related parameters are listed in the Table $\mathbf{1}$.

Table 1: Fixed model parameters used in this simulation

Density $\rho 7900 \mathrm{~kg} / \mathrm{m}^{3}$

Particle radius $r 125 \mu \mathrm{m}$

Coefficient of restitution

crestitution 0.3

Coefficient of static

friction cfriction 0.5

Coefficient of rolling

friction crolling 0.5

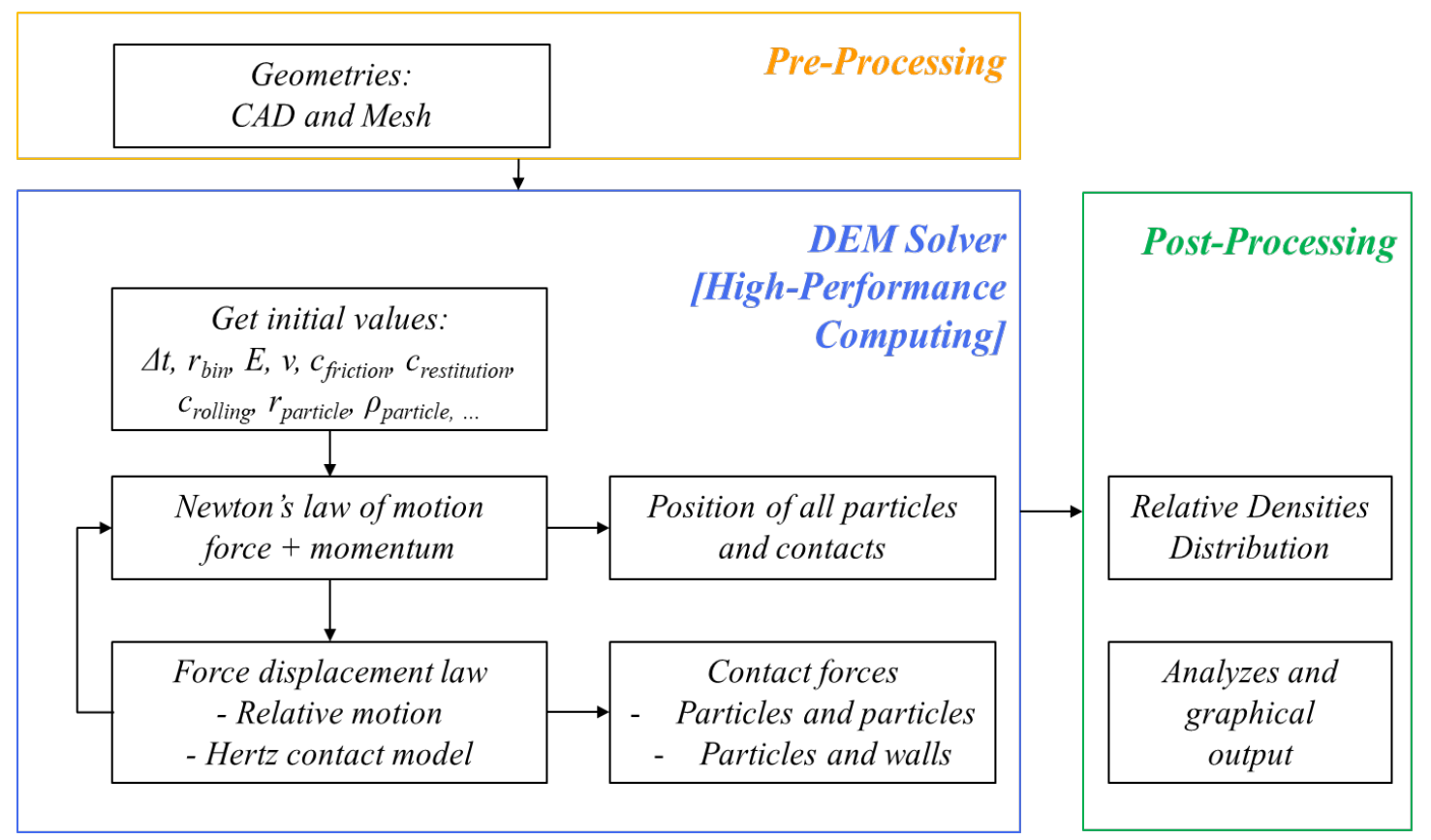

Figure 4: DEM Modelling Approach for determination of initial density distribution.

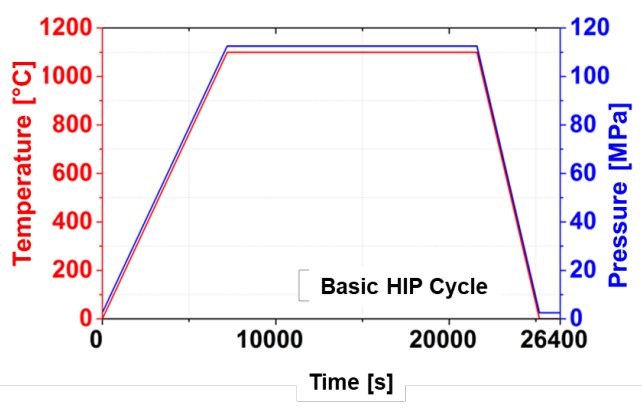

Figure 5: Temperature and pressure profile for the HIP densification process
A DEM simulation is divided to three different steps (Figure 4): model design by preprocessing, numerical solution of Newton's equation of motion, export and evaluation by post-processing. After the DEM simulation, the positions of the particles and the contact forces between individual particles as well as between particles and the capsule are included in the output data. 


\section{Coupled process simulation of powder filling via DEM and powder densification via FEM}

The determined relative densities via DEM after the powder filling process were mapped into the FEM model, which was used to simulate the powder densification process. The basic HIP cycle was applied to the densification process (Figure 5). The HIP densification model (capsule material SS304, powder material SS316L) based on FEM was preliminarily implemented in the user- defined material model (UMAT) ${ }^{[9]}$, which is used to simulate the material behaviours in ABAQUS (Figure 6). The initial values, e.g. stress, strain increment, relative densities, were generated and calculated in ABAQUS. Other mechanical properties depending on the temperature were calculated in the UMAT. The time independent plasticity model of Kuhn and Downey ${ }^{[10,11]}$ and the rate dependent plasticity (viscoplasticity) model of Abouaf ${ }^{[12]}$ were coupled in a combined model. After the calculation of the inelastic strain according to this combined model, the stresses and relative densities were updated at the end of each increment. In this way, the calculation continues until the end of the densification process.

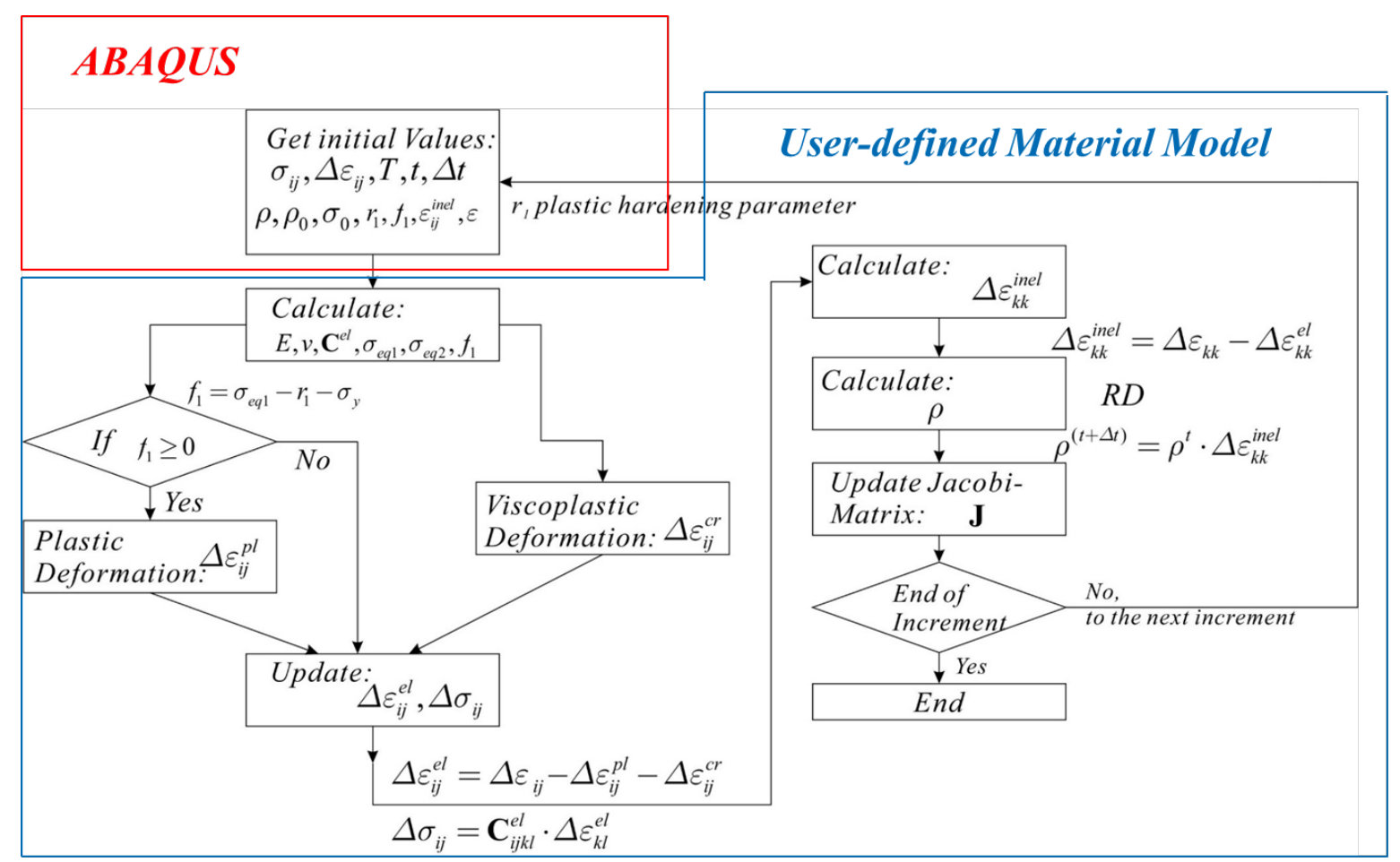

Figure 6: Flow chart of HIP densification model based on FEM

\section{Results and Discussion}

Figure 7 shows a numerical determination of the density distribution in a cylindrical capsule, which was also experimentally determined as shown in Figure 3. With decreased defined powder particle size, the amount of powder particle increases exponentially that leads to the increasing computational time. Before the size of the particle for the simulation completely fixed, the sensitivity analyse was conducted on a small capsule using four discrete particle sizes $(r=60 \mu \mathrm{m}$, $125 \mu \mathrm{m}, 250 \mu \mathrm{m}, 500 \mu \mathrm{m}$ ). The radius of $125 \mu \mathrm{m}$ was defined for the simulation, because the simulation using $r=125 \mu \mathrm{m}$ results to a relative small size effect compared to the simulation with size of $r=60 \mu \mathrm{m}$, meanwhile, the simulation with large particle size can be dramatically reduced. The rheological material properties of SS316L powder relevant for the powder filling simulation 
were determined and calibrated using a normal flow test with measured angle of repose and a powder flowmeter test with measured filling time.

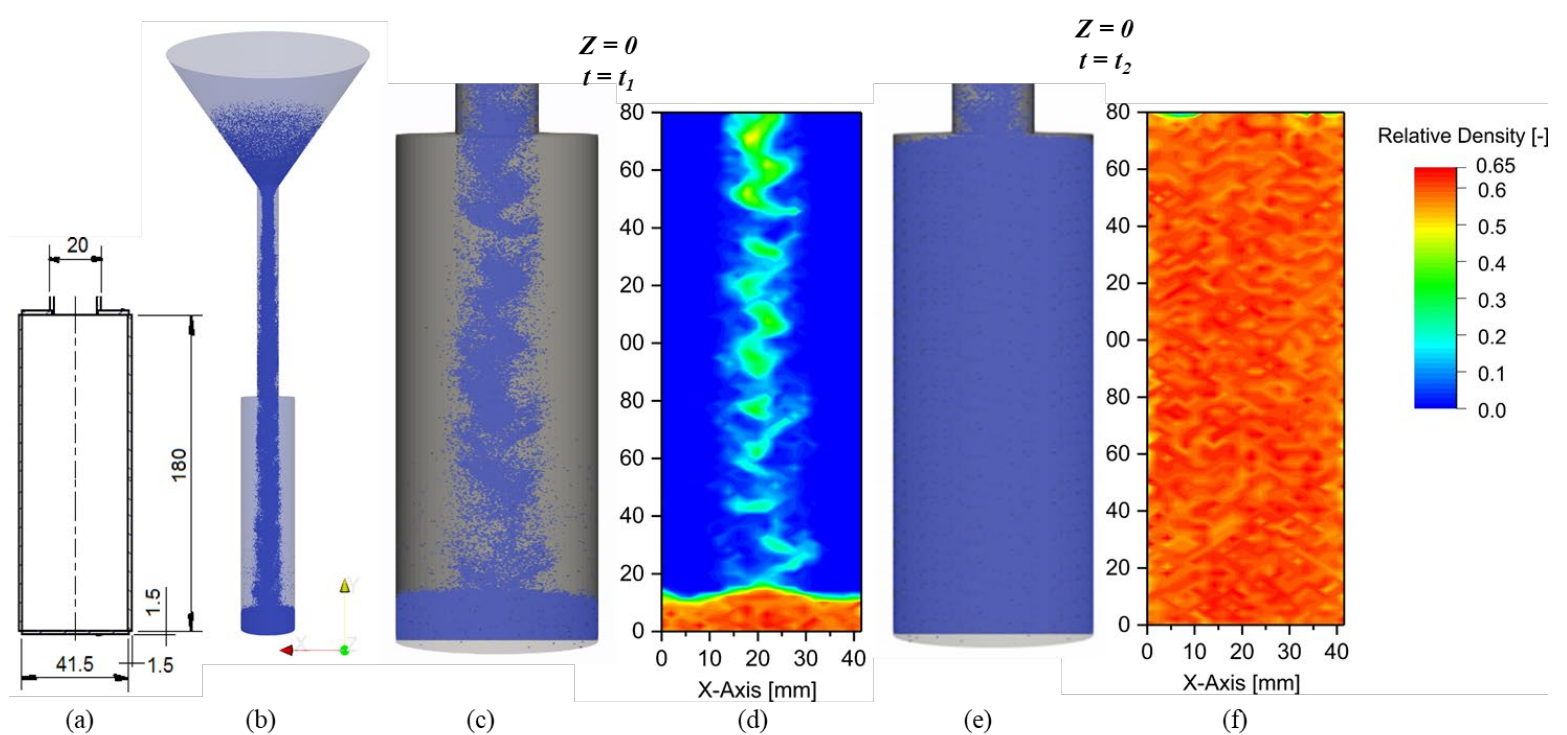

(a)

(b)

(c)

(e)

(f)

Figure 7: Filling process using DEM Simulation: (a) capsule geometry, (b) visualization of the filling process, (c) visualization and (d) exported density distribution of the cross section at intermediate step, (e) visualization and ( $f$ ) exported density distribution of the cross section at the filling end.

In this case, only mono-sized powder particles with a radius of $125 \mu \mathrm{m}$ were considered in the simulation. After calibration and validation, the material properties were implemented to simulate the powder filling process in a cylindrical capsule (Figure 7(a)) using a full 3-D DEM model Pre-consolidation processes such as vibration or tapping have not yet been considered. The powder was inserted firstly in a funnel and then flowed into the capsule (Figure 7(b)). The cross-sectional view of the plane $\mathrm{Z}=0$ in intermediate steps during filling are shown in Figure 7(c) and (e). With in-house written scripts, the real-time relative density distributions (Figure 7(d) and (f)) can be obtained. Three-dimensional density distribution was exported to FEM simulation model in which the next processing step is simulated.

The density distributions determined via DEM and "Image Analysis" were mapped in the FEM simulation models (Figure 8 (a) and (b)), respectively. After the capsule filling without any further pre-consolidation process, a high-density region was observed in the middle of the capsule, whereas the positions close to the capsule wall were measured with relatively low filling densities. The difference of the determined densities is $~ 2 \%$ in average. The averaged densities inside the capsule measured by "Image Analysis" and simulated by DEM were 60\% and 58\%, respectively. For the capsule filling process, only mono-sized powder particles were considered in the simulation. The density distribution might be close to the real case, while the real particle size distribution of the powder material was considered in the simulation.

A script was written and implemented in ABAQUS to map the local densities into the discretized finite element model. Three-dimensional density database was imported into the simulation model prior to HIP, and the densities of the meshes on the cross section is shown in Figure 9, which was determined via DEM simulation. 
The FEM models (Figure 8 (a) and (b)) were subsequently used to calculate the HIP densification process. The contours of the final shapes were compared as shown in Figure 8 (c). The contour of the final shape with initial relative density input from DEM simulation is smaller than that with the initial relative density input from "Image Analyse". The largest dimension difference of $3.8 \%$ was observed around the top corners of the capsule, possibly due to the difference of the determined density distribution based on two different methods. This deviation can be reduced by improving the prediction with the consideration of particle size distribution via DEM simulation.

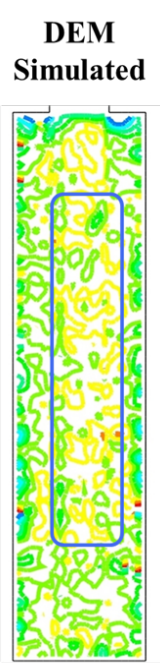

Initial Rel

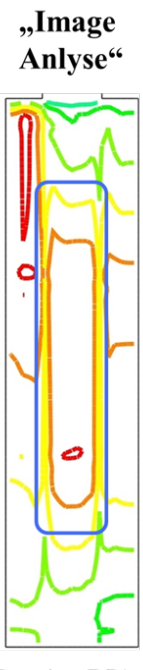

(b)

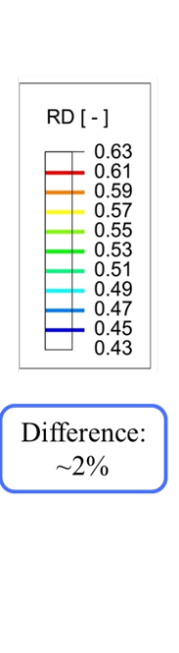

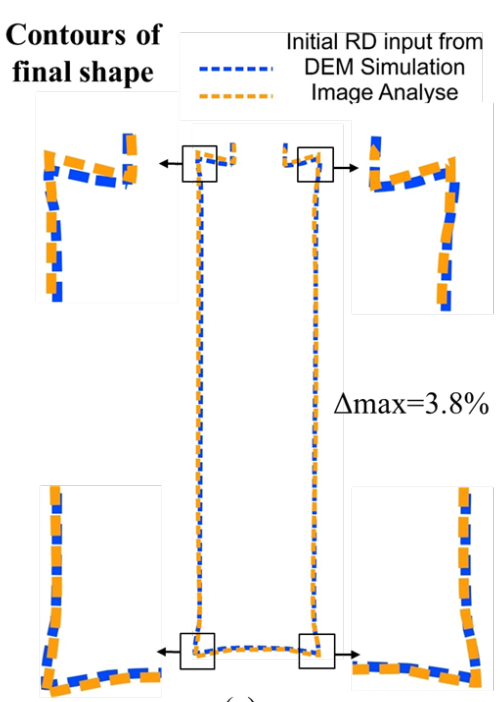

(c)

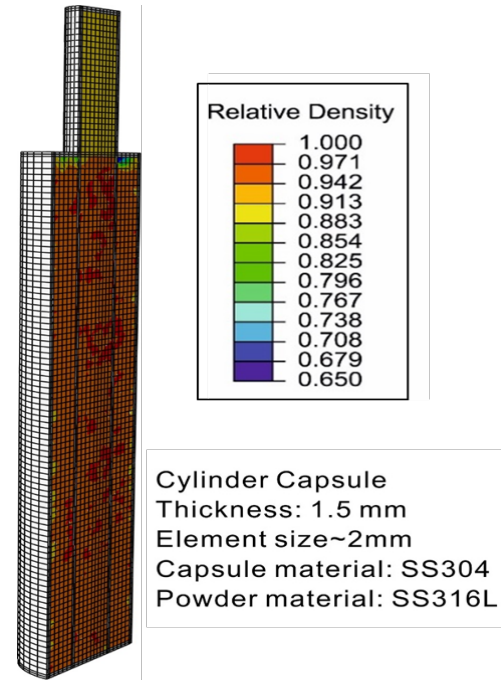

Figure 9: Relative densities determined via DEM were assigned into the simulation model prior to HIP, which was cut on the cross section.

\section{Conclusions and Outlook}

In this work, the simulation approach with coupled DEM and FEM modelling has been firstly used to calculate the capsule filling densities and predict the densification behaviour of powder materials during HIP Processes. To predict the powder density distribution inside capsule prior to HIP and the final geometry after HIP precisely, several influencing factors, capsule materials and powder properties, have been considered. The simulation results of the density distribution correspond well with the experimental measurement, which renders the effectiveness of this approach in the precise prediction of the component final shape. To improve the precision of this approach, other factors, such as particle size distribution, different kind of pre-densification processes, will be considered in the following work. Additionally, more complex shaped capsules will also be studied in the future.

\section{Acknowledgement}

This work was performed under the support from the RWTH Aachen University HPC Compute Project No. rwth0248. 


\section{References}

[1] H. V. Atkinson, S. Davies, Metall and Mat Trans A 2000, 31, 2981. https://doi.org/10.1007/s11661-000-0078-2

[2] E. Olevsky, S. van Dyck, L. Froyen, L. Delaey (Eds.: F. H. Froes, J. Hebeisen, R. Widmer) 1996.

[3] L. J. R. Trasorras, M. E. Canga, W. B. Eisen, Proc. of the Int. Conf. on Powder Metallurgy \& Particulate Materials 1994.

[4] T. Shiokawa, Y. Yamamoto, S. Hirayama and Y. Nagamachi, in Proc. of the Int. Conf. on Hot Isostatic Pressing (Ed.: K. Ishizaki) 1992, p. 225.

[5] M. Abouaf, G. Raisson and E. Wey, in Proc. of the 3rd Int. Conf. on Isostatic Pressing 1986, p. 10.

[6] C. van Nguyen, Numerical Simulation of Hot Isostatic Pressing with Particular Consideration of Powder Density Distribution and Temperature Gradient, Shaker Verlag 2016.

[7] C. van Nguyen, A. Bezold, C. Broeckmann, Powder Metallurgy 2014, 57, 295. https://doi.org/10.1179/1743290114Y.0000000087

[8] C. Kloss, C. Goniva, Supplemental Proceedings: Materials Fabrication, Properties, Characterization, and Modeling, Volume 2011.

[9] C. van Nguyen, Y. Deng, A. Bezold, C. Broeckmann, Computer Methods in Applied Mechanics and Engineering 2017, 315, 302. https://doi.org/10.1016/j.cma.2016.10.033

[10]L. T. Kuhn, R. M. McMeeking, International Journal of Mechanical Sciences 1992, 34, 563. https://doi.org/10.1016/0020-7403(92)90031-B

[11]H. A. Kuhn, A. Lawley, G. E. Dieter, Powder metallurgy processing: new techniques and analyses, Academic Press 1978.

[12] M. Abouaf, J. L. Chenot, G. Raisson, P. Bauduin, Int. J. Numer. Meth. Engng. 1988, 25, 191. https://doi.org/10.1002/nme.1620250116 